\title{
Acesso à sala de vacinas nos serviços de atenção primária à saúde*
}

\author{
Ariana Vitalina Ferreira ${ }^{1}$, Clara Fonseca Oliveira ${ }^{2}$, Eliete Albano de Azevedo Guimarães ${ }^{3}$, \\ Ricardo Bezerra Cavalcante ${ }^{4}$, Juliano Teixeira Moraes ${ }^{5}$, Valeria Conceição de Oliveira ${ }^{6}$
}

\footnotetext{
* Este artigo é resultado da Dissertação de Mestrado da Universidade Federal de São João del-Rei, Campus Centro-Oeste Dona Lindu, pesquisa financiada Fundação de Amparo à Pesquisa do Estado de Minas Gerais (FAPEMIG 002/2014/Prope).

${ }^{1}$ Enfermeira, Mestre em Enfermagem. Enfermeira da Prefeitura Municipal de Divinópolis. Divinópolis, MG, Brasil. E-mail: arianaeju@hotmail.com.

${ }^{2}$ Discente do curso de Graduação em Enfermagem da Universidade Federal de São João del-Rei, Campus Centro-Oeste Dona Lindu. Divinópolis, MG, Brasil. E-mail: clarinha_fo@yahoo.com.br.

${ }^{3}$ Enfermeira, Doutora em Ciências da Saúde. Professora Adjunto da Universidade Federal de São João del-Rei. Divinópolis, MG, Brasil. Email: elietealbano@ufsj.edu.br.

${ }^{4}$ Enfermeiro, Doutor em Ciência da Informação. Professor Adjunto da Universidade Federal de São João del-Rei. Divinópolis, MG, Brasil. E-mail: ricardocavalcante@ufsj.edu.br.

${ }^{5}$ Enfermeiro. Doutorado em Ciências Aplicadas à Saúde do Adulto. Professor Adjunto da Universidade Federal de São João del-Rei. Divinópolis, MG, Brasil. E-mail: julianotmoraes@hotmail.com.

${ }^{6}$ Enfermeira. Doutora em Enfermagem em Saúde Pública. Professora Adjunto da da Universidade Federal de São João del-Rei. Divinópolis, MG, Brasil. E-mail: valeriaoliveira@ufsj.edu.br.
}

Recebido: 21/07/2016.

Aceito: 31/03/2017.

Publicado: 25/08/2017.

Como citar esse artigo: Ferreira AV, Oliveira CF, Guimarães EAA, Cavalcante RB, Moraes JT, Oliveira VC. Acesso à sala de vacinas nos serviços de atenção primária à saúde. Rev. Eletr. Enf. [Internet]. 2017 [acesso em:_____ ] 19:a31. Disponivel em: http://dx.doi.org/10.5216/ree.v19.42468.

\section{RESUMO}

Objetivou-se analisar o acesso às salas de vacinas nas Estratégias Saúde da Família de um município de Minas Gerais/Brasil. Trata-se de um estudo de caso único, qualitativo, realizado no ano de 2015 . As salas de vacinas foram as unidades de análise do estudo. Realizadas entrevistas semiestruturadas com 49 usuários e 30 profissionais de saúde e observação da rotina de atendimento em sala de vacina. Procedeu-se a técnica de análise de conteúdo temática, com as categorias pré-estabelecidas. Os dados demonstraram que o acesso às salas de vacina na atenção primária à saúde apresenta entraves, implicando na exclusão de usuários ao serviço de imunização, por isso a importância de conhecer as dificuldades do acesso ao serviço, a fim de reorganizá-lo de forma a abranger a vacinação a todo público-alvo do Programa Nacional de Imunização.

Descritores: Acesso aos Serviços de Saúde; Vacinas; Atenção Primária à Saúde; Enfermagem em Saúde Comunitária.

\section{INTRODUÇÃO}

A imunização é uma ação comprovada para controlar e eliminar as doenças infecciosas e estima-se que mais de 30 doses de vacina são administradas globalmente a cada segundo e nenhuma outra intervenção de saúde atinge tantas pessoas, ou é capaz de impedir uma gama tão variada de problemas de saúde pública(1).

O Programa Nacional de Imunizações (PNI) do Brasil é considerado como um dos programas mais completos dentre as nações em desenvolvimento, com a distribuição de mais de 300 milhões de doses anuais entre os 44 imunobiológicos, nas 35.000 salas de 
vacinação, aproximadamente, espalhadas em todo território com o objetivo de ofertar de forma universal vacinas a todos os grupos etários ${ }^{(2)}$. Apesar dos esforços empregados pelo PNI e as estratégias de vacinação adotadas para eliminar diversas doenças e controlar outras, existe a necessidade de identificar fatores que influenciam as coberturas vacinais ${ }^{(3)}$, que apresentam-se heterogêneas, principalmente com disparidade dessas coberturas nos municípios, o que pode comprometer o controle, eliminação ou erradicação das doenças imunopreveníveis ${ }^{(3-5)}$.

A Estratégia Saúde da Família (ESF) por ter a delimitação da área de abrangência, o número previsto de famílias sob sua responsabilidade, o diagnóstico da situação de saúde e ações definidas, diferenciam do atendimento das unidades de saúde tradicionais, devendo portanto, facilitar o acesso universal aos serviços de saúde, um dos princípios preconizados pelo $S_{U} S^{(6)}$. Dada a relevância e o impacto indiscutível que a imunização exerce na saúde das populações, é fundamental a condução de estudos que visam o conhecimento e elucidação dos fatores que facilitam ou dificultam o acesso às salas de vacinas nas unidades de saúde do SUS.

O termo acesso é utilizado como utilização do serviço e implica em identificar barreiras, sob quaisquer aspectos, que possam impedir o indivíduo de obter um direito ou um serviço que the é garantido formalmente $^{(7)}$. Pode ser resultado da combinação de diversos fatores, de dimensões distintas, que se interrelacionam, definidas como de ordem geográfica e sócio-organizacional ${ }^{(8)}$, socioeconômico e cultural ${ }^{(9)}$. $\mathrm{Na}$ literatura consultada não encontramos estudos sobre acesso à sala de vacina em nosso meio. Questionase, assim: como a organização da Estratégia Saúde da Família tem influenciado o acesso à sala de vacinas?

O objetivo deste trabalho, portanto, foi analisar o acesso às salas de vacinas nas Estratégias Saúde da Família de um município da região ampliada de saúde Oeste de Minas Gerais.

\section{MÉTODO}

\section{Caracterização do caso}

Foi realizado estudo de caso único em um município de pequeno porte do Estado de Minas Gerais, com cobertura de $100 \%$ de ESF. Trata-se de um município reconhecido nacionalmente como o segundo polo moveleiro do Brasil ${ }^{(10)}$.

A partir de 1999 o município ampliou a rede de serviços à saúde, tendo, como meta, a reorganização da atenção primária com a ESF, e hoje existem nove unidades de estratégias de saúde da família - ESF com diversidade de território por se tratar de equipes urbanas e duas rurais. Possuem diferenças na produção social da saúde, pois as equipes rurais são itinerantes, apresentando situações dificultadoras, como a distância percorrida pelos profissionais e os obstáculos das estradas não pavimentadas.

\section{Elaboração do modelo teórico}

A coleta de dados foi precedida pela elaboração de um modelo teórico (Figura 1), incorporando as 
dimensões e critérios relacionados à acessibilidade em sala de vacinas, alicerçada no referencial teórico de Donabedian $^{(8)}$ e Fekete ${ }^{(9)}$ nas seguintes dimensões do acesso: organizacional, geográfico, sociocultural e econômico.

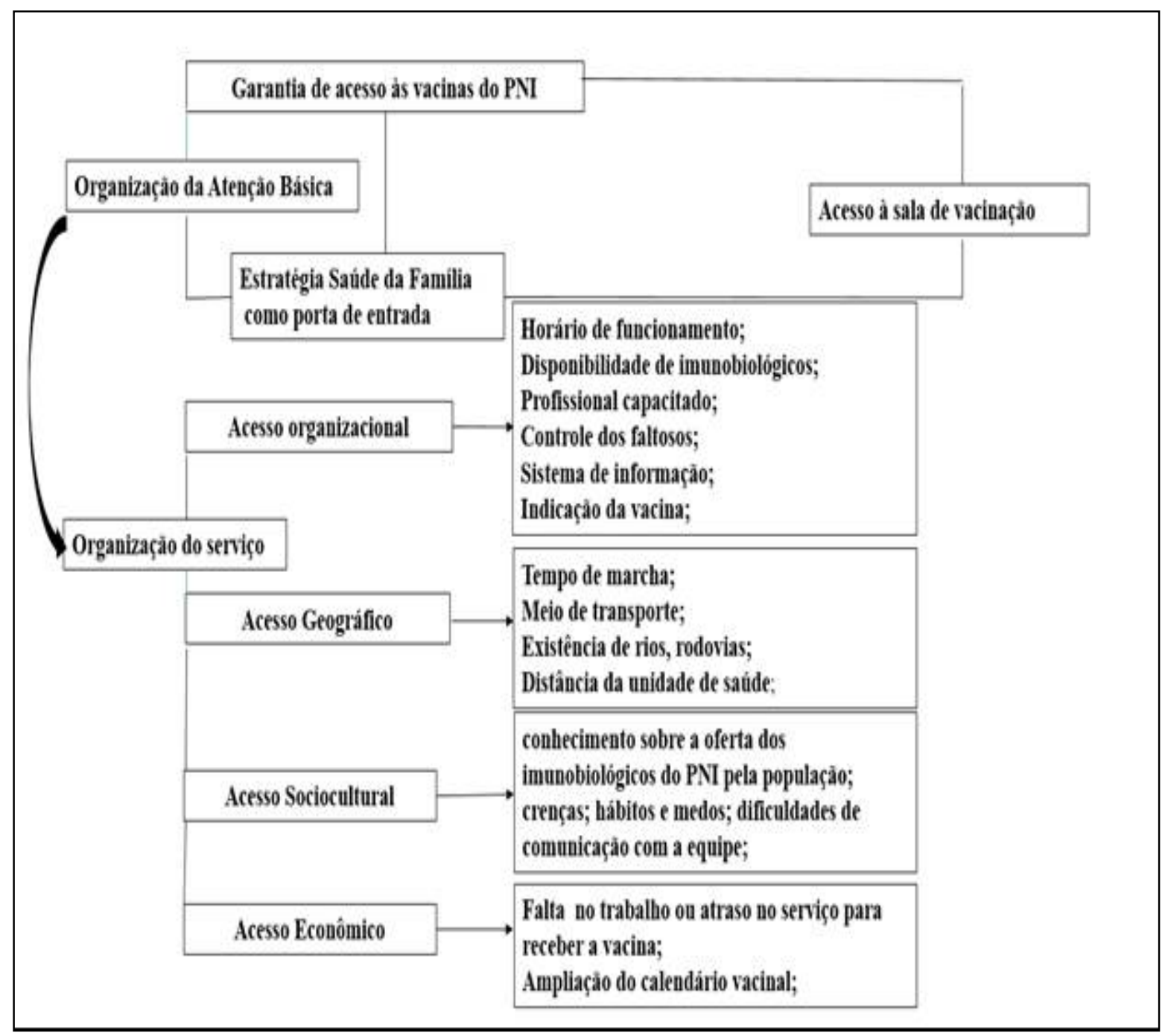

Figura 1: Modelo teórico para a análise do acesso às salas de vacina na Atenção Primária à Saúde.

Para cada uma das dimensões da acessibilidade descritas no modelo, foram definidos os aspectos relacionados à sala de vacinas levando-se em consideração a interação e a influência desses no acesso à sala de vacinação.

Na dimensão organizacional, foram definidos os itens: horário de funcionamento; disponibilidade dos imunobiológicos; profissional capacitado; controle dos faltosos; sistema de informação e indicação da vacina. Já na dimensão geográfica do acesso foi estabelecido: o tempo de marcha; o meio de transporte; a existência de rios, rodovias e a distância da unidade de saúde. O acesso sociocultural foi caracterizado pelo conhecimento sobre a oferta dos imunobiológicos do PNI pela população; crenças, hábitos, medos e dificuldades de comunicação com a equipe. E a dimensão econômica do acesso definiu a falta ou atraso no trabalho para receber a vacina e a ampliação do calendário vacinal.

Neste estudo, para a análise do acesso às salas de vacina da atenção primária, os termos acesso e 
acessibilidade foram utilizados como sinônimos.

Coleta e análise dos dados

Os participantes desta pesquisa foram trabalhadores das equipes da ESF entre médicos, enfermeiros, auxiliares/técnicos de enfermagem, agente comunitário de saúde e a coordenadora da atenção primária, em um total de 31 profissionais entrevistados, identificados com a letra (P) seguidos da numeração das entrevistas. Como critério de inclusão, estabeleceu-se a atuação de, no mínimo, seis meses de serviço na APS para que o profissional conhecesse a realidade de sua unidade. Dos 53 entrevistados possíveis, 23 profissionais estavam em férias, licença saúde, afastamentos ou o tempo de serviço na ESF era menor do que seis meses. Então, restaram 30 possíveis participantes, que após aceitarem participar voluntariamente da pesquisa, mediante assinatura do Termo de Consentimento Livre e Esclarecido, foram todos entrevistados.

Além dos profissionais, participaram da pesquisa 49 usuários referentes ao público-alvo de vacinação: mãe de criança, adolescente, gestante, adulto/trabalhador, idoso e acamados com capacidade cognitiva preservada, identificados com a letra (U) seguidos na numeração da entrevista. Foram realizadas entrevistas com usuários que aguardavam atendimento de saúde na unidade, procurou-se alcançar todo o público-alvo, realizando visitas domiciliares para os grupos não encontrados na unidade. A indicação das visitas domiciliares foi feita pela equipe de saúde juntamente com a pesquisadora.

Tendo como critério para o encerramento das entrevistas no mínimo um usuário para cada grupo do público-alvo do PNI em cada sala de vacina e todos os profissionais de saúde que se enquadravam no critério de inclusão.

A pesquisa de campo, foi realizada no período de dois de março a 13 de maio de 2015, teve por base um levantamento de dados primários por meio de observação direta de natureza descritiva, realizada em todas as ESF. O registro dessas observações foi feito em um diário de campo elaborado após cada período, identificada como "notas de observação" (NO).

Os dados foram analisados segundo a técnica de análise de conteúdo temática descrita por Bardin ${ }^{(11)}$, sendo as categorias pré-definidas nas quatro dimensões do acesso: geográfico, organizacional, sociocultural e econômico. Esta forma de categorização chamada de "caixas" é aplicável no caso de a organização do material decorrer diretamente dos fundamentos teóricos hipotéticos ${ }^{(11)}$. Na etapa da exploração do material, utilizou-se o software Atlas.ti versão 7.5.6 para auxiliar no processo de codificação e recorte das unidades de registro e contexto.

O projeto foi aprovado pelo Comitê de Ética em Pesquisa da Universidade Federal de São João DelRei/Campus Centro Oeste, sob o parecer no 910.125. CAAE: 37653214.7.0000.5545.

\section{RESULTADOS}

\section{A dimensão organizacional do acesso}

O principal entrave do acesso em relação à dimensão organizacional foi a falta do imunobiológico, 
também apontada em outros estudos ${ }^{(5,12-13)}$ e constitui um importante obstáculo para que se atinja uma adequada cobertura vacinal.

[...] a última vez que eu fui lá foi na semana passada, foi para vacinar a minha filha da vacina de três meses, mas não tinha a vacina, porque a menina que foi trazer a vacina disse que não tinha chegado no posto de $C$, eles pegam lá e trazem para cá no posto do $S$, falou para eu voltar na próxima semana que já teria a vacina [...] U1.

A indisponibilidade de vacinas contribui para baixas coberturas ou atraso do calendário vacinal. Nos Estados Unidos em 2009, durante a escassez de vacina Hib, houve uma recomendação provisória para adiar a dose de reforço, mas manter o esquema primário, esta orientação resultou na diminuição de $66 \%$ para $39,5 \%$ de crianças com calendário vacinal completo ${ }^{(14)}$.

No Brasil, é importante ressaltar que, durante a coleta de dados deste estudo, o país enfrentava um desabastecimento de vacinas em virtude de repasse insuficiente pelo Ministério da Saúde devido reformas nos laboratórios produtores. Entretanto, foi observado a rotina do agendamento de vacinas, independente, da escassez do imunobiológico.

[...] Tem vacina que dá na terça-feira, para não desperdiçar, eu tenho vacina que dá na sexta-feira. Aí a gente olha e se tiver no dia certo a gente chama a enfermeira ou a técnica que faz a vacina. E se não for o dia, a gente orienta a pessoa e explica o porquê [...] P58.

O receio pelo desperdício coloca os trabalhadores de saúde em situação difícil entre decidir pela abertura do frasco de multidoses e a perda das doses não utilizadas ou agendamento para outro dia, quando há um grupo maior, evitando o desperdício, mas induzindo perdas de oportunidades vacinais, e aumentado o risco de adoecimento ${ }^{(15)}$.

Nos registros do diário de campo, resultado da observação direta nos serviços, foi possível identificar que o horário de funcionamento não é o mesmo, existe sala de vacinas que não atende no horário de almoço e encerra suas atividades mais cedo, além da disponibilidade de vacinas em dias e horários específicos. Em outras, a unidade apesar de aberta, não realiza vacinas no horário de almoço e há concentração de imunobiológicos em determinados dias.

[..] Vindo no horário do funcionamento, que é de sete às cinco, tem este horário até cinco horas que o pessoal que trabalha é bem tranquilo e tem o horário de almoço também, que continua funcionando a sala de vacina direitinho [...] P25.

[...] De sete às quatro, menos no horário do almoço, essa uma hora fica sem ninguém, ou um técnico ou enfermeira na unidade, mas o pessoal já está bem orientado quanto a isso [...] P61.

Mesmo após mais de duas décadas de SUS, a dificuldade de acesso se configura como um dos principais problemas na APS/ESF que não oferecem disseminadamente acesso fácil a seus usuários e nem dispõem de tecnologias organizacionais consolidadas para tal ${ }^{(16)}$. A sala de vacina que funciona em horário comercial e, normalmente, não oferece horários alternativos limita o acesso, principalmente para aquelas pessoas inseridas no mercado de trabalho ${ }^{(14)}$. 
No que tange as oportunidades de vacinação, uma única estratégia não é suficiente para melhorar as taxas de imunização e diminuir as dificuldades de acesso. As vacinas precisam ser acessíveis em todas as oportunidades possíveis e todos os esforços devem ser feitos para uma ampla divulgação dos imunobiológicos disponíveis ${ }^{(14)}$.

Alguns usuários demonstraram insatisfação pela espera prolongada. A fala da usuária relata que após longa espera foi embora sem receber a vacina.

[...] eu fiquei lá, ninguém me falou nada se iria aplicar ou se não iria, cheguei sete hora, deu sete, sete e meia, oito, eu pegava serviço oito horas, oito e quinze eu vim embora, eu não vou ficar esperando mais não, ninguém não deu a solução de nada [...] U52.

Determinados profissionais demonstraram-se sobrecarregados com os atendimentos realizados para a população da área de abrangência, precisam realizar outras atividades e não apenas à vacinação (NO). Foram detectados, através da observação e também referidos pelos entrevistados que as dificuldades em relação ao tempo de espera são vivenciadas em unidades de saúde com número maior de pessoas cadastradas (NO).

[...] Número de funcionários, porque as meninas estão sobrecarregadas, esse PSF aqui ele atende gente demais e o número de funcionários é muito pequeno, então, na minha opinião, não sei se é porque eu vim de Belo Horizonte e lá o número de funcionários nos postos é praticamente o dobro daqui e assim atende muito menos pessoas que o PSF daqui $[\ldots] \cup 23$.

A ESF é composta por equipe multiprofissional que atua em área geográfica definida e população adscrita, responsabilizando-se por uma população com média de 3.000 pessoas, e máximo de 4.000 pessoas, podendo ser menor de acordo com o risco e a vulnerabilidade apresentados ${ }^{(6)}$. A grande extensão territorial do país e suas particularidades demográficas e geográficas, assinalam para a necessidade de redução dos usuários por equipe da ESF, para que se consiga oferecer um acesso efetivo ao cuidado ${ }^{(16)}$.

\section{A dimensão geográfica do acesso}

No caso do município estudado, as características da organização geográfica destacam-se pelo grande número de comunidades rurais e a escassez de transporte urbano nessas comunidades. $\mathrm{O}$ acesso nesta dimensão não sofreu críticas por parte dos usuários que residem na área urbana do município, o que já era esperado, uma vez que as unidades de saúde em foco estão localizadas no território onde as famílias a elas cadastradas residem. Entretanto, os profissionais e usuários da zona rural, entrevistados, identificaram barreiras ao acesso à sala de vacina.

[...] até porque nós não temos ônibus, não temos veículos públicos que vão a esses locais com horário correto, então, largar os afazeres da roça para vir aqui é difícil, eles dependem às vezes de uma carona ou o próprio patrão que vem para a cidade, isso não impossibilita, mas é um grande dificultador! [...] P49.

Em comparação com residentes de áreas urbanas, moradores das áreas rurais necessitam de uma 
viagem mais longa em face de outros problemas, tais como pior manutenção das estradas e dificuldades de transporte público. Verificou-se que as formas de deslocamento são em sua maioria a condução a pé, a cavalo e a bicicleta. Os moradores da zona rural são os que mais enfrentam dificuldades de acesso até a unidade, como retrata a fala de um profissional.

[...] Em zona rural o acesso tanto de distribuição geográfica e acesso de chegar até a unidade é difícil porque na nossa comunidade eu tenho casas que ficam perto de I, para o paciente vir aqui às vezes ele não tem carro, não tem transporte dentro da comunidade, então ele vem a pé, ele vem a cavalo, então, nessa questão de acesso à unidade, estar chegando até aqui é difícil [...] P75.

Constitui em grande desafio o acesso geográfico, o que inclui peculiaridades que favorecem ou impedem as pessoas de receber os cuidados em sala de vacinas. A acessibilidade geográfica não se define apenas pela distância da casa das pessoas até a unidade de saúde, mas também pelas barreiras geográficas encontradas no seu deslocamento, como a existência de rios, morros, estradas de terra, alagamentos e a inexistência de transporte público ${ }^{(9)}$. O Brasil conta hoje com as ESF Ribeirinhas e as Unidades Básicas de Saúde Fluviais, levando a equipe de saúde a locais de difícil acesso, como a região da Amazônia e do Mato Grosso do Sul ${ }^{(6)}$.

É necessário um maior esforço e criatividade na tentativa de se programar a oferta da vacinação, com uma periodicidade regulada, tendo como objetivos a promoção da equidade e a garantia do acesso à saúde como direito $^{(5,17)}$.

\section{A dimensão sociocultural do acesso}

A acessibilidade cultural, na perspectiva da população, está relacionada dentre outras coisas, ao conhecimento sobre a oferta dos serviços de saúde, as dificuldades de comunicação com a equipe, aos hábitos e crenças $^{(9)}$.

A não vacinação está relacionada ao medo da dor, à falta de conhecimento, aos costumes e crenças que a sociedade traz juntamente com seus valores culturais ${ }^{(18)}$. A atividade de vacinar parece ser moralmente inquestionável, sendo reconhecida como um dos maiores avanços da ciência no controle e erradicação das doenças infectocontagiosas. Entretanto, é preciso respeitar as posições morais, individuais e culturais existentes $^{(18)}$.

Em algumas entrevistas, são expressas as falas dos usuários e dos profissionais sobre a não vacinação devido às práticas integrativas e complementares. Uma usuária observa a vacinação como uma agressão ao corpo e considera um absurdo a vacinação contra o vírus HPV.

[...] eu acho um absurdo a vacina da gripe! Sabe, eu acho uma ofensa à pessoa, criança não precisa de jeito nenhum! É como se eu tivesse agredindo fisicamente meus filhos, outra vacina é a HPV para as meninas, que absurdo, eu surto quando eu escuto falar que irão vacinar as meninas de HPV, eu acho que tem que haver a educação [...] U37.

[...] Um dificultador também é a homeopatia, porque o homeopata fala que não é para vacinar algumas vacinas, mas a gente conversa, orienta[...] E2. 
Os grupos mais difíceis de alcançar são aqueles com baixa escolaridade ou falta de entendimento sobre a imunização ou aqueles que culturalmente são contrários às vacinas ${ }^{(19)}$. Existem movimentos antivacinas que podem influenciar negativamente nas taxas de coberturas vacinais. Assim, é fundamental, no contexto atual, um diálogo aberto com os pais e indivíduos avessos à vacinação, oferecendo informações precisas sobre as doenças e os eventos adversos das vacinas ${ }^{(14)}$.

Em relação aos hábitos e costumes da população, este estudo identificou como um obstáculo no dia a dia do serviço de imunização, a falta de preservação do cartão de vacina pelo adulto e o hábito de procurar a vacinação somente quando ocorre um ferimento ou quando é exigido o cartão vacinal completo, para ingressar no serviço em determinadas empresas. Esse fato aumenta o risco de eventos adversos e gastos públicos com doses de vacinas administradas desnecessariamente.

[...] geralmente os adultos só vêm quando precisam do cartão de vacina em dia! Quando machuca, vem procurar a vacina antitetânica e não pensam em prevenir antes [...] P26.

[...] uma dificuldade que eu tenho é o do cartão, o paciente fala que perdeu e você tem que fazer tudo de novo, ou não lembra, tem que fazer um esquema. Então eu acho que a primeira coisa que a gente deveria fazer é conscientizar da importância do cartão [...] P36.

O desconhecimento da situação vacinal da população, ainda é um dos maiores problemas que se enfrentam, pois o indivíduo não costuma preservar seu comprovante de vacinação ${ }^{(20)}$ o que pode resultar em vários problemas como a dificuldade em proporcionar ao profissional o acesso à informação. É importante continuar o esquema de vacinação que se iniciou em oportunidades anteriores e a complexidade do calendário de vacinação pode afetar a confiabilidade de uma história de vacinação verbal ${ }^{(21)}$. As vacinas pertencem a um grupo de produtos biológicos, com excelente perfil de segurança, entretanto, não são isentas de eventos adversos. Um estudo de coorte retrospectivo para examinar o risco de reações locais, depois da administração da vacina dupla adulto, encontrou aumento do risco em relação ao número de doses administradas previamente ${ }^{(22)}$. Por isso a apresentação do comprovante vacinal é imprescindível para a continuidade dos esquemas, evitando doses desnecessárias de vacina e risco de eventos adversos.

\section{A dimensão do acesso econômico}

Nos últimos tempos, verificou-se uma grande mobilização internacional por novas vacinas e campanhas vacinais ${ }^{(15)}$, proporcionando como resultado no Brasil a ampliação do calendário vacinal do PNI, como: as vacinas tetraviral, hepatite $A$, tríplice bacteriana acelular, a vacina contra o papilomavírus e o oferecimento da vacina contra a hepatite B para todas as faixas etárias ${ }^{(23)}$. Esse acesso ampliado e gratuito às vacinas é visto com otimismo pela sociedade, que agora não precisa recorrer a clínicas particulares para garantir a imunização.

[...] a minha menina sempre foi vacinada no posto, eu nunca paguei uma vacina, não tive essa necessidade, porque o posto atendeu a todas as demandas de vacina [...] U64. 
A ampliação do calendário vacinal foi reforçado pelo profissional de saúde, que relata a quantidade de vacinas incluídas no PNI nos últimos tempos e cita como exemplo as vacinas contra pneumonia, meningite e varicela.

[...] Olha para você ver a quantidade de vacina que a gente tem aqui, e chegou pelo SUS. A gente tinha aqui antes a Pólio, a Tríplice, Sarampo isolado, não tinha Hepatite B, não tinha Pneumonia, não tinha Meningite, não tinha varicela. [...] P3.

Entretanto, o acesso aos serviços de saúde é influenciado pela condição econômica e social das pessoas $^{(24)}$. Observou-se inquietude dos pais, sem condições financeira para oferecer a vacina a seus filhos que estão fora da faixa etária preconizada pelo PNI.

[...] não concordo da faixa etária que eles colocam de algumas vacinas, por exemplo, a campanha da gripe, eu fiquei na fila de espera, esperando sobrar vacina pra vacinar o meu menino porque ele não estava dentro da faixa etária e hoje eu não tenho condições pra comprar a vacina [...] U56.

\section{CONSIDERAÇÕES FINAIS}

A vacinação, por ser considerado um dos serviços fundamentais da APS, não poderia impor barreiras ao acesso, porém, neste estudo foram enumeradas e discutidas dificuldades encontradas pelos profissionais de saúde e os usuários ao utilizarem esse serviço. Observou-se que muitos entrevistados relataram dificuldades em relação a elementos organizativos dos serviços, como a centralização em dia e horário específico para vacinar e o horário de funcionamento da sala de vacina. Para os moradores da zona rural é difícil o acesso geográfico e o hábito da não preservação do cartão pelo adulto foi um dos grandes entraves no acesso sociocultural.

Percebe-se, também, nos resultados desse estudo, a importância da equipe de enfermagem no acesso à vacinação; profissionais capacitados e atualizados diminuem as perdas de oportunidades vacinais.

Diante dessas considerações, é importante lembrar que o município estudado possui $100 \%$ de cobertura da ESF e apresentou barreiras ao acesso, desse modo, levanta o questionamento de como está o acesso à vacinação nos municípios com cobertura inferior, fazendo necessários novos estudos sobre o tema ainda pouco estudado.

\section{Agradecimentos}

À Fundação de Amparo à pesquisa do Estado de Minas Gerais/FAPEMIG no fornecimento de apoio financeiro para realização da pesquisa.

\section{REFERÊNCIAS}

1. Berkley S. Make vaccine coverage a key UN health indicator. Nature [Internet]. 2015 [acesso em: 22 ago. 2017];526(7572):165. Disponível em: http://dx.doi.org/10.1038/526165a. 
2. Ministério da Saúde, Secretaria de Vigilância em Saúde, Departamento de Vigilância das Doenças Transmissíveis. Manual de normas e procedimentos para vacinação [Internet]. Brasília: Ministério da Saúde; 2014 [acesso em: 22 ago. 2017]. 176 p. Disponível em: http://bvsms.saude.gov.br/bvs/publicacoes/manual_procedimentos_vacinacao.pdf.

3. Queiroz LLC, Monteiro SG, Mochel EG, Veras MASM, Sousa FGM, Bezerra MLM et al . Cobertura vacinal do esquema básico para o primeiro ano de vida nas capitais do Nordeste brasileiro. Cad Saude Publica [Internet]. 2013 [acesso em: 22 ago. 2017];29(2):294-302. Disponível em: http://dx.doi.org/10.1590/S0102-311X2013000200016.

4. Domingues CMAS, Teixeira AMS. Coberturas vacinais e doenças imunopreveníveis no Brasil no período 1982-2012: avanços e desafios do Programa Nacional de Imunizações. Epidemiol. Serv. Saúde [Internet]. 2013 [acesso em: 22 ago. 2017];22(1):9-27. Disponível em: http://dx.doi.org/10.5123/S1679-49742013000100002.

5. Santos GRD, Silva SS, Guimarães EAA, Cavalcante RB, Oliveira VC. Avaliação do monitoramento rápido de coberturas vacinais na Região Ampliada de Saúde Oeste de Minas Gerais, 2012. Epidemiol. Serv. Saúde [Internet]. 2016 [acesso em: 22 ago. 2017];25(1):55-64. Disponível em: http://dx.doi.org/10.5123/S1679-49742016000100006.

6. Ministério da Saúde, Secretaria de Atenção à Saúde, Departamento de Atenção Básica. Política Nacional de Atenção Básica [Internet]. Brasília: Ministério da Saúde; 2012 [acesso em: 22 ago. 2017]. 110 p. Disponível em:

http://189.28.128.100/dab/docs/publicacoes/geral/pnab.pdf.

7. Starfield B. Atenção primária: equilíbrio entre necessidades de saúde, serviços e tecnologia [Internet]. Brasília: Ministério da Saúde; 2002 [acesso em: 22 ago. 2017]. Disponível em:

http://www.dominiopublico.gov.br/download/texto/ue000039.pdf.

8. Donabedian A. Los espacios de lasalud: aspectos fundamentales de laorganización de laatención médica. México: Fondo de Cultura Económica; 1988. 772 p.

9. Fekete MC. Estudo da acessibilidade na avaliação dos serviços de saúde. In: Santana JP, Santos I, Fekete MC, Galvão EA, Mandelli MJ, Penna MLF et al., organizadores. Desenvolvimento gerencial de unidades básicas do Sistema Unico de Saúde (SUS): programa de desenvolvimento de recursos humanos. Brasília: Organização Pan-Americana da Saúde; 1997. p. 177-84.

10. Programa das Nações Unidas para o Desenvolvimento; Instituto de Pesquisa e Econômia Aplicada; Fundação João Pinheiro. O índice de desenvolvimento humano municipal brasileiro. Atlas do Desenvolvimento Humano no Brasil 2013 [Internet]. Brasília: PNUD; 2013 [acesso em 22 ago. 2017]. Disponível em:

http://atlasbrasil.org.br/2013/data/rawData/publicacao_atlas_municipal_pt.pdf.

11. Bardin L. Análise de conteúdo. Lisboa: Edições 70; 2011.

12. Barrera L, Trumbo SP, Bravo-Alcántara P, Velandia-González M, Danovaro-Holliday MC. From the parents' perspective: a user-satisfaction survey of immunization services in Guatemala. BMC Public Health [Internet]. 2014 [acesso em: 22 ago. 2017];14:231. Disponível em: http://dx.doi.org/10.1186/1471-2458-14-231.

13. Lopes EG, Martins CBG, Lima FCA, Gaíva MAM. Situação vacinal de recém-nascidos de risco e dificuldades vivenciadas pelas mães. Rev Bras Enferm [Internet]. 2013 [acesso em: 22 ago. 2017];66(3):338-44. Disponível em: http://dx.doi.org/10.1590/S0034-71672013000300006.

14. Sabnis SS, Conway JH. Overcoming Challenges to Childhood Immunizations Status. Pediatr Clin North Am [Internet]. 2015 [acesso em: 22 ago. 2017];62(5):1093-109. Disponível em:

http://dx.doi.org/10.1016/j.pcl.2015.05.004.

15. Stokes-Prindle C, Privor-Dumm L, Haidari L, Connor D, Wateska A, Broowm S et al. Coverage, cost, and safety impacts of primary container choice [Internet]. Baltimore: Johns Hopkins Bloomberg School of Public Health; 2013 [acesso em: 22 ago. 2017]. Disponível em: http://www.jhsph.edu/research/centers-and-institutes/ivac/projects/IVAC PrimaryContainerBriefReport-2013.pdf.

16. Tesser CD, Norman AH. Repensando o acesso ao cuidado na Estratégia Saúde da Família. Saude soc. [Internet]. 2014 [acesso em: 22 ago. 2017];23(3):869-83. Disponível em: http://dx.doi.org/10.1590/S0104-12902014000300011. 17. Pereira BFB, Martins MAS, Barbosa TLA, Silva CSO, Gomes LMX. Motivos que levaram as gestantes a não se vacinarem contra H1N1. Cien Saude Colet [Internet]. 2013 [acesso em: 22 ago. 2017];18(6):1745-52. Disponível em: http://dx.doi.org/10.1590/S1413-81232013000600025.

18. Lessa SC, Schramm FR. Proteção individual versus proteção coletiva: análise bioética do programa nacional de vacinação infantil em massa. Cien Saude Colet [Internet]. 2015 [acesso em: 22 ago. 2017];20(1):115-24. Disponível em: http://dx.doi.org/10.1590/1413-81232014201.14882013.

19. Print F. Accessing hard to reach groups, travelers and looked after children. Hum Vaccin Immunother [Internet]. 2013 [acesso em: 22 ago. 2017];9(6):1372-3. Disponível em: http://dx.doi.org/10.4161/hv.24693.

Rev. Eletr. Enf. [Internet]. 2017 [acesso em:_____];19:a31. Disponível em: http://dx.doi.org/10.5216/ree.v19.42468. 
20. Lemos EO, Pedrosa DR, Raniéri PSG, Pires CAA, Queiroz AM. Avaliação do cumprimento do calendário de vacinação dos adolescentes de uma escola municipal. Adolescência \& Saúde [Internet] 2013 [acesso em: 22 ago. 2017];10(2):23-9. Disponível em: http://www.adolescenciaesaude.com/detalhe_artigo.asp?id=365.

21. Cutts FT, Izurieta HS, Rhoda DA. Measuring coverage in $\mathrm{MNCH}$ : design, implementation, and interpretation challenges associated with tracking vaccination coverage using household surveys. PLoS Med [Internet] 2013 [acesso em: 22 ago. 2017];10(5):e1001404. Disponível em: http://dx.doi.org/10.1371/journal.pmed.1001404.

22. Jackson LA, Yu O, Nelson J, Belongia EA, Hambidge SJ, Baxter R et al. Risk of medically attended local reactions following diphtheria toxoid containing vaccines in adolescents and young adults: a Vaccine Safety Datalink study. Vaccine [Internet]. 2009 [acesso em: 22 ago. 2017];27(36):4912-6. Disponível em:

http://dx.doi.org/10.1016/j.vaccine.2009.06.038.

23. Ministério da Saúde, Secretaria de Vigilância em Saúde, Departamento de Vigilância das Doenças Transmissíveis. Nota Informativa no 149, de 2015/CGPNI/DEVIT/SVS/MS. Informo as mudanças no Calendário Nacional de Vacinação para o ano de 2016 [Internet]. Brasília: Ministério da Saúde; 2015 [acesso em: 22 ago. 2017]. Disponível em: http://www.saude.pi.gov.br/uploads/warning_document/file/129/Nota_Informativa_149.pdf.

24. Tonhá ACM, Souza Júnior PRB, Bezerra ALQ, Santos TAP, Rosso CFW. Acesso aos serviços de saúde nos municípios do entorno sul do Distrito Federal. Rev. Eletr. Enf. [Internet]. 2015 [acesso em: 22 ago. 2017];17(2):238-46. Disponível em: https://doi.org/10.5216/ree.v17i2.29422. 